\title{
La palude di sangue. Mito e tragedia in Pavese
}

\author{
Marco Antonio Bazzocchi \\ Università degli Studi di Bologna \\ marco.bazzocchi@unibo.it
}

\section{Riassunto}

Il saggio studia l'argomento della palude nell'opera di Pavese. L'immagine della palude costituisce un ricco archetipo che mette in rilievo tutta la sua forza ermeneutica dei Dialoghi con Leucò. La palude è lo spazio del sangue e delle origini della vita. Le memorie di questo spazio rimangono incise nell'immaginario umano. Pavese usa ancora quest'idea nei suoi romanzi e racconti, specialmente in Il diavolo sulla collina, dove la palude fa riferimento alla vita in stato primordiale. Questo ancestrale ricordo vive ancora nel mondo della media borghesia, creandone un rapporto chiaramente conflittuale.

Parole chiave: Palude, sangue, simbolo, immagine, archetipo, memoria, vita originaria, Pavese, Dialoghi con Leucò, Il diavolo sulla collina.

The bloody marshland. Myth and tragedy in Pavese

\begin{abstract}
The essay investigates the theme of the marshland in Pavese's works. The imagery of the marshland has an archetypical value, that shows itself with a great evidence in the Dialoghi con Leuco. The marshland is the bloody place of the origin of the life. The memory of this place remains imprinted in the human's mind. Pavese reuses this idea also in his novels and tales, especially in the Il diavolo sulla collina, where the marshland refers to the primordial life. This memory survives in the middle class' world and shocks it inside.
\end{abstract}

Key words: Marshland, blood, symbol, imagine, arquetype, memory, original life, Pavese, Dialoghi con Leucò, Il diavolo sulla collina.

Bazzocchi, Marco. 2011. La palude di sangue. Mito e tragedia in Pavese. Cuadernos de Filología Italiana, $\mathrm{n}^{\mathrm{o}}$ extraordinario: págs. 49-60. 
Il racconto Lo zio acquatico apre la sezione Evoluti e mutanti delle Cosmicomiche di Italo Calvino. Viene affrontato il momento dell'evoluzione dai pesci ai rettili, che lo scrittore mette in scena come contrasto tra le scelte del giovane Qfwfq (pesce mutante verso la condizione di rettile) e il prozio N'ba N'ga (abbarbicato al suo mondo di vecchio pesce). Come avviene in tutta la raccolta, Calvino racconta alcuni passaggi epocali attraverso la prospettiva straniante di un personaggio astratto che di volta in volta sta dalla parte del futuro in contrasto con altri personaggi che invece rifiutano il cambiamento. Epoche lontanissime della storia umana vengono ripercorse così attraverso un filtro comico che permette di collocare il mito in una fase regressiva, mentre Qfwfq (voce narrante) si pone dalla parte dell'evoluzione e della razionalità. Qui sceglie di abbandonare le acque melmose dove è nato e di sperimentare la nuova vita sulla terra, utilizzando le pinne come zampe. Invece il vecchio e saggio prozio resta nell'acqua della laguna, fedele a una forma di vita umida al posto della secca e scomoda terra. Le ragioni del prozio sono molto chiare, e hanno una loro giustificazione. Mentre le terre emerse rappresentano il rischio dell'adattamento, la necessità di una modificazione che può portare alla perdita della propria identità, nell'acqua «la vita si sarebbe conservata così come s'era svolta fin qui, nelle sue forme piene e perfette, senza metamorfosi o aggiunte di dubbio esito, e ognuna avrebbe potuto approfondire la propria natura, arrivare all'essenza di sé e di ogni cosa». Anche la giovane Lll, di cui Qfwfq è innamorato, si convincerà e abbandonerà la vita sulla terra per regredire, insieme allo zio acquatico, alla condizione di pesce.

Calvino usa il racconto per proporre una sua idea del mito e del tempo. Alla perfezione delle forme stabili del passato oppone l'imperfezione del futuro e il rischio dell'ibridazione. La palude dove lo zio attira la giovane Lll è il luogo dell'origine, e come tale è fisso nel tempo, senza possibilità di mutamento. Attraverso il filtro comico e meditativo (una versione moderna dell'umorismo romantico) il narratore afferma invece l'esigenza dell'evoluzione. Qfwfq affronta il destino come apertura del possibile, pur consapevole dei privilegi del passato. Il passato, e le figure che vi rimangono attaccate (in particolare le figure femminili) conservano il fascino dell'anomalia (lo zio è visto esplicitamente come un mostro) e insieme della compiutezza.

L'acqua paludosa dei pesci del Carbonifero può avere come modello vicino le paludi dove si mescolano gli elementi della Piccola cosmogonia portatile di Raymond Queneau. Ma di sicuro Calvino ha nella memoria un'immagine che percorre i Dialoghi con Leucò di Pavese, quella della palude Boibeide.

Cercherò innanzitutto di capire la funzione di quest'immagine nell'opera di Pavese.

Boibeide viene nominata e descritta per la prima volta nel dialogo Le Muse (30 gennaio-1 febbraio 1946), destinato poi a chiudere la raccolta nell'assetto definitivo. Il dialogo si svolge tra il poeta Esiodo e Mnemòsine, madre delle Muse, sul monte Elicona, anche se fin dall'inizio il poeta evoca tempi primordiali in cui la dea aveva altri nomi (lui stesso la chiama Melete, uno dei nomi originari delle muse quando erano ancora tre) e abitava su monti più impervi, in Tracia o in Tessaglia. Il centro del dialogo è occupato dalla spiegazione di Mnemòsine intorno al valore 
degli «attimi estatici», cioè degli attimi in cui il riconoscimento improvviso rende felice la vita umana. Parla Mnemòsine: «Tu guardavi l'ulivo, l'ulivo sul viottolo che hai percorso ogni giorno per anni, e viene il giorno che il fastidio ti lascia, e tu carezzi il vecchio tronco con lo sguardo, quasi fosse un amico ritrovato e ti dicesse proprio la sola parola che il tuo cuore attendeva» (Pavese 1968a: 165). Secondo la dea la vita degli uomini può conformarsi, o perlomeno tendere, a questo modello di compiutezza. Esiodo invece controbatte che il tempo mortale non può assumere tali caratteristiche, perché la ripetizione implica la noia, il fastidio: «Ma gli istanti mortali non sono una vita. Se io volessi ripeterli perderebbero il fiore. Torna sempre il fastidio». Allora Mnemòsine vuole convincere Esiodo della possibilità di far partecipare gli uomini al divino e utilizza il ricordo della palude Boibeide, per la quale Pavese in un appunto rimanda a «Properzio (Fasti)» (Pavese 1968a: 182), ma che in realtà si trova nominata spesso in Esiodo (lo vedremo).

La palude è il luogo dell'origine e della continuità, il luogo da cui provengono uomini, mostri e dèi: «Una landa nebbiosa di fango e di canne, com'era al principio dei tempi, in un silenzio gorgogliante. Generò mostri e déi di escremento e di sangue. Oggi ancora i Téssali ne parlano appena. Non la mutano né tempo né stagioni. Nessuna voce vi giunge» (Pavese 1968a: 166). Secondo la Musa, questa è la strada attraverso cui l'uomo si congiunge con il mostruoso e con il divino, «ogni uomo nasce in quella palude di sangue», e quindi ogni uomo può conoscere il sacro e il divino, quel sacro e quel divino che porta nella memoria.

Pavese riesce con il dialogo a ricomporre i tre aspetti dell'esistere che si presentano in forme diverse nell'intera opera ${ }^{1}$. Con le parole di Mnemòsine, la palude originaria diventa il luogo di un'unica radice che accomuna mostri, uomini e dèi. Si tratta quindi di un punto capitale per l'interpretazione dei Dialoghi, ma - come cercherò di dimostrare - un simbolo che ha una funzione centrale in tutta l'ultima parte dell'opera di Pavese. Quello che in tutti i dialoghi precedenti sembrava vivere in forme separate qui invece ritrova unità, o perlomeno un'ipotesi di unità, la memoria dell'unità, nell'insegnamento della Musa a Esiodo. E non a caso Esiodo è il poeta della Teogonia, cioè dell'opera dedicata alla genealogia divina che Pavese traduce presumibilmente tra il 1947 e il '48, a dialoghi conclusi. Il riferimento a Properzio contiene invece un errore, probabilmente di memoria, perché il poeta latino non ha mai scritto Fasti ma solo Elegie.

Pavese però ha trovato il nome di Properzio, questo è sicuro, in una nota del saggio di Károly Kerényi sulla Kore, il saggio che fa parte del famoso volume JungKerényi, Prolegomeni allo studio scientifico della mitologia (il libro esce nella Collana viola nella traduzione di Angelo Brelich nel 1948, ma di sicuro Pavese lo conosce già prima). Qui infatti, nella nota $5 \mathrm{a} \mathrm{p.} 205$ viene esplicitamente indicato un verso di Properzio, dal libro secondo delle Elegie $(2,11)$ dove si trova il riferimento al lago di Boibeis in Tessaglia, il luogo dove avvengono le nozze tra la divinità nominata Brimo e Zeus. Brimo - spiega Kerényi - è una figura di divinità arcai-

${ }^{1}$ Cfr. Frontaloni (2005: 101): «L'intero corpo dei Dialoghi si svolge, così, come un lungo e tormentato discorso intorno al momento di passaggio fra ordinamento pre-olimpico e ordinamento olimpico». 
ca che comprendeva in sé sia Demeter che Persefone (madre e figlia) ma anche Hekate: «essa è Demeter, Persefone e Hekate in una sola persona». Si tratta di una forma della Kore primitiva, alla quale si può far risalire anche Artemis. Dunque Pavese trova illustrato in Kerényi uno degli aspetti più interessanti della mitologia greca arcaica, cioè la compresenza in una stessa figura archetipica di figure che poi avranno evoluzioni diverse. Ma la spiegazione di Kerényi dice di più. Nell'arcaico culto dei misteri di Eleusi infatti Brimo partorisce un figlio, cioè un fanciullo divino.

Questo figlio non viene definito, «egli non è che la creatura, il frutto». Ecco le parole di Kerényi che possono aver attirato l'attenzione di Pavese: «L'idea complessiva della nascita, del sempre rinnovato inizio della vita, congiunge madre figlia e fanciullo in un'unità significativa. Il senso della nascita in questo caso non è l'inizio assoluto, non è il primo e l'unico cominciamento, bensì la continuità nell'ininterrotta serie delle nascite. Nell'identità di madre e figlia, la madre, la sempre di nuovo partoriente, nella cui figura e nel cui destino entra l'iniziato, appare come un essere perpetuo; il fanciullo è il segno, che questa perpetuità è superindividuale, continuazione e continua rinascita nella posterità» (Kerényi 1971: 206).

Le parole di Kerényi portano Pavese a concepire un paradigma della continuità. Il lago Boibeis è luogo di violenza (le nozze persefonee) ma anche di nascita, di una nascita superindividuale, per cui l'individuo si ricongiunge alla sua posterità. E infatti lo scrittore, nella introduzione al dialogo Le Muse, allude al fatto di aver scelto una sola figura femminile a rappresentare le nove, o tre, o due, Muse. Ma aggiunge, elitticamente, «In questo mondo che trattiamo, le madri sono sovente le figlie» (Pavese 1968a: 166). Cita chiaramente, senza dirlo, il saggio di Kerényi, e aggiunge la sua musa alla famiglia di Kore. Ma l'ultima battuta di Melete-Mnemòsine spiega proprio la continuità della vita e la persistenza dell'origine nella vita di ogni uomo: «Non capisci che l'uomo, ogni uomo, nasce in quella palude di sangue? E che il sacro e il divino accompagnano anche voi, dentro il letto, sul campo, davanti alla fiamma? Ogni gesto che fate ripete un modello divino. Giorno e notte, non avete un istante, nemmeno il più futile, che non sgorghi dal silenzio delle origini» (Pavese 1968a: 166). La palude Boibeide, la palude di sangue, è iscritta nella memoria dell'individuo. È la continuità che lega l'individuo all'origine e al futuro. Non a caso è la dea della memoria a parlare con Esiodo.

La natura sanguinosa e selvaggia di questa origine va però indagata.

In una lettera a Mario Untesteiner del 7 maggio 1948 Pavese ritorna esplicitamente alla mitologia tessala e rivela la fonte della sua interpretazione di Esiodo parlando della Thessalische Mythologie di Paula Philippson, studio che verrà tradotto da Angelo Brelich l'anno successivo nella collana viola col titolo Origine e forme del mito greco. Come afferma Pavese stesso, il libro sta alla base del dialogo Le cavalle (25-26 febbraio 1947).

Cerchiamo di capire cos'è nell'interpretazione della Philippson la palude Boibeide e perché questo luogo abbia attirato per la seconda volta e con tale intensità lo scrittore.

La Philippson indaga l'espansione della religione olimpica di Zeus e la formazione del suo cosmo, «in seguito alla quale decadono gli esseri primordiali, Chaos, 
Gaia, Uranos, Pontos» (Philippson 1949 : 133). Questo complesso fenomeno ha due aspetti: uno storico, che si può seguire proprio in Tessaglia, e uno mitico che si può ricostruire attraverso la Teogonia di Esiodo. Così abbiamo l'intersezione di due temporalità diverse: la prima è il tempo storico lineare, la seconda il tempo mitico, «il cui carattere comporta che in esso un avvenimento si compia una sola volta e tuttavia si compia sempre e sia un presente continuo». Credo che questo modello di temporalità doppia abbia influito con molta forza sul pensiero di Pavese, molto di più del modello temporale che di solito si ricollega a Proust o a Thomas Mann, due autori che comunque Pavese ammira. E credo anche che sia necessario partire di qui per capire la particolare intersezione nell'opera di Pavese di forme apparentemente astratte come i dialoghi mitologici e forme narrative più specifiche come i romanzi, senza dimenticare i passaggi intermedi dei racconti contenuti in Feria d'agosto.

Ma è la stessa descrizione della Tessaglia condotta dalla Philippson che non può averlo lasciato indifferente: la collina di Pherai, luogo di culto di una divinità arcaica femminile che prende lo stesso nome, domina una pianura ai limiti della quale si stagliano il Pelion, l'Ossa e l'Olimpo, i monti sacri dove cammina il nuovo dio vincitore, Zeus. Ai piedi del Pelion si trova il lago Boibe, dove pascolano i cavalli e i tori nei quali si incarnano forme diverse del divino (la Philippson spiega che «gli dei ellenici non sono né umani né animaleschi. Ma la figura umana e la figura animale sono forme d'epifania, nelle quali quelle divinità si davano a conoscere ... in quella forma di conoscenza che è il mito» (Philippson 1949: 122). La dea Pherai, figlia di Admeto signore delle profondità, qui sulle rive del lago entra in relazione con Enodios ctonio (Hermes). E qui nasce Asklepios che «molto prima di diventare figlio di Apollo e dio professionale dei medici, era stato un grande dio ctònio della Tessaglia» (Philippson 1949 : 138). Madre di Asklepios è Koronis, «che bagnava i piedi nel lago di Boibe». Ma nello stesso tempo a Koronis risale anche Hermes nel suo aspetto di nutritore (Trophonios), quindi Hermes è originariamente identico a Asklepios «e la comune origine ctònia conferisce a entrambi gli dèi l'attributo del serpente attorcigliato intorno a una verga». La grande dea di Pherai appare così come un concentrato simbolico di aspetti divini che vanno dal primordiale all'olimpico, dallo ctonio all'uranico. Ma la divinità, portatrice di aspetti oscuri e inferi, subisce una trasformazione nel momento in cui entra nel cosmo dello Zeus olimpico, e di questa trasformazione testimonia proprio l'opera di Esiodo: «il primo che presentò questo mito come una visione comprendente in forma genealogica l'intero mondo nella sua unità, fu il grande poeta beotico.

Di notte, sul pendio dell'Elicona, sulla cui cima Poseidon Hippios aveva la sua sorgente sacra, le Muse gli concessero questa rivelazione» (Philippson 1949: 185). Esiodo è il creatore di un'opera che raccoglie le tracce del passato più arcaico e le sistematizza nel nuovo ordine cosmico. La sua genealogia ci mostra come il cosmo, cioè la fase ordinata del mondo, si formi come unità per mezzo dell'incontro di due opposti. È la forma polare del pensiero greco, per cui i contrari di una coppia non sono solo collegati tra loro ma sono condizionati alla loro opposizione. Ogni singola divinità greca si presenta in questa forma polare.

Se analizziamo il dialogo Le cavalle vediamo che tutto quanto qui riassunto assume un valore ermeneutico fondamentale. Bisogna anche ricordare che la stesu- 
ra del dialogo venne preceduta da quella dell'Ospite, ispirata chiaramente alla lettura dei riti cruenti di rigenerazione che Pavese trovava nel Ramo d'oro di Frazer. Qui è Eracle a assumere il ruolo di eroe civilizzatore che diffonde la nuova legge degli dèi olimpici, gli dèi che stanno appartati sul monte e che rifiutano l'antica tradizione dei sacrifici umani. La leggenda di Litierse che uccide gli stranieri per fecondare le sue terre si ricollega in realtà, come spiega Frazer, a un ulteriore aspetto del rapporto tra Demetra e Persefone, cioè alla sparizione del grano sottoterra per rinascere come spiga. E non a caso Pavese sottolinea la componente materna connessa al mito. Litierse parla esplicitamente di una divinità femminile definita «la terra, la Madre, la Grotta» che attende i sacrifici di sangue.

Nelle Cavalle dialogano Ermete e Chirone, il centauro, al quale viene affidato il piccolo Asclepio, salvato da Apollo dalle fiamme che hanno ucciso la madre, Coronide. Il nucleo mitologico si trova ancora una volta nella Philippson: «....anche Asklepios, il potente dio della Tessaglia, che suo padre Apollo aveva strappato dal corpo morente della bella Koronis, cresce in un prato davanti alla veneranda grotta di Chirone» (Philippson 1949: 218).

Apparentemente anche qui si contrappongono due modi, quello nuovo degli dèi olimpici rappresentati da Ermete e quello arcaico rappresentato da Chirone. In realtà le due epoche sono legate, e infatti Chirone chiama Ermete col nome Enodio, che risale come abbiamo visto $\mathrm{a}$ una forma più primitiva della divinità. $\mathrm{E}$ gli ricorda che ambedue derivano da una radice bestiale, originati dalla palude di sangue: «Lo siamo bestiali. E proprio tu, Enodio, che a Làrissa eri coglia di toro, e all'inizio dei tempi ti sei congiunto nel fango della palude con tutto quanto di sanguigno e ancora informe c'era al mondo, proprio tu ti stupisci?» (Pavese 1968a: 27-28). Ma Ermete dichiara che quei tempi sono ormai lontani, e che lui stesso ora ha funzioni diverse. La morte di Coronide è connessa proprio a questa trasformazione dei tempi. Lo spiega Chirone che si erge a difensore del passato, quando «la bestia e il pantano eran terra d'incontro di uomini e dèi» (Pavese 1968a: 28). L'autore ci indica così che un sostrato arcaico persiste a tutte le variazioni avvenute con la comparsa degli dèi olimpici. In realtà il modello mitico che lui conosce implica che questo passaggio cronologico non abbia azzerato la persistenza dell' arcaico. Corònide, donna e cavalla, aveva in sé la memoria della palude, la traccia della sua origine bestiale, «l'informe furore sanguigno che l'aveva generata». E per questo Apollo l'ha uccisa, dal momento che gli dèi nuovi distruggono tutto ciò che porta in sé i segni del caos originario: «Ogni volta che il caos trabocca alla luce, alla loro luce, devon trafiggere e distruggere e rifare» (Pavese 1968a: 29).

Si delinea così una tensione di forze all'interno dei dialoghi, o perlomeno di molti tra loro. Una tensione che può essere vista come il modo in cui Pavese interpreta il contrasto nietzschiano tra Apollo e Dioniso, che ha però ora un fondamento diverso. Ma è anche chiara la propensione di Pavese per la realtà primitiva e titanica a scapito dell'età olimpica. Lo dichiara lui stesso nel Mestiere di vivere: «L'età titanica (mostruosa e aurea) è quella di uomini-mostri-dèi indifferenziati. Tu consideri la realtà come sempre titanica, cioè come caos umano-divino (mostruoso), ch'è la forma perenne della vita» (24 febbraio 1947; Pavese 2000: 326). Mi sembra che «forma perenne» sia una chiara accettazione da parte di Pavese dell'idea di un 
tempo del mito come tempo della persistenza. L'evento si compie una volta per sempre ed è sempre presente. L'età titanica, cioè l'età del caos di umano e divino, cioè del mostruoso, non è finita.

Ma nei dialoghi non tutti i personaggi si pongono nello stesso atteggiamento di fronte alla palude di sangue. Ci sono anzi personaggi che aprono una prospettiva esterna a quella del titanismo arcaico. E allora può essere la lettura di un altro libro sull'antichità classica, La fisiologia del mito di Mario Untesteiner, ad aver orientato Pavese. Untesteiner spiega infatti che anche gli dèi omerici vedono limitata la loro azione dalla presenza della morte: «Contro la morte nulla essi possono fare [...] Catastrofe, interruzione, limitazione sono tutte forme della morte, e la morte costituisce il più alto significato del destino, di Moira, che è un originario dèmone appartenente alla religiosità preellenica e perciò del tutto distinto dagli dèi olimpii» (Untersteiner 1972: 117). Gli dèi conoscono Moira ma nello stesso tempo le sono sottoposti. Hanno anch'essi un limite iscritto nel loro destino, ma nella prospettiva dell'uomo sono essi a incarnare questo stesso limite. E allora a Moira si sostituisce dàimon. Gli uomini vedono in dàimon la presenza del limite, di quel limite che "prostra l'azione etica di un uomo e che dall'uomo può essere conosciuta prima del suo attuarsi» (Untersteiner 1972: 119). In altri termini, dàimon è il modo in cui il divino passa nell'umano creando contraddizioni e dissidi. È il concetto che regola il rapporto tra dèi e uomini, o meglio il riflesso della divinità sull'individuo. La volontà umana può contrapporsi al dàimon che è nell'uomo e generare così un contrasto all'interno dell'individuo. Dàimon diventa un rapporto tra libertà individuale e necessità, un rapporto che iscrive la vita dell'individuo nel tragico: «Le contraddizioni del mondo, rivelate dai contrasti tra gli dèi, sono penetrate ben addentro nella coscienza umana. [...] In dàimon la pluralità contraddittoria degli dèi, rimanendo ineliminata, si concilia con l'unità, entro la sfera della coscienza umana: la spiritualità della religione olimpica si innesta nel naturalismo dei Mediterranei. Tutti i fermenti del mito si sono polarizzati nell'interesse profano dell'umana creatura» (Untersteiner 1972: 120). Già Guido Guglielmi aveva indicato il nucleo tragico della contrapposizione come binomio esistenziale : «Destino è libertà e scacco della libertà» (Guglielmi 1998: 132).

La spiegazione di Untersteiner è fondamentale per capire l'intreccio di mito e tragedia che percorre tutti i dialoghi di Pavese, ma apre una prospettiva anche sull'intera esperienza narrativa dell'autore. Alla persistenza del mito e dell'arcaico (il mostruoso-selvaggio) si sovrappone l'apertura del tempo tragico, cioè la possibilità data all'uomo di modificare il proprio destino. La morte, come esito catastrofico, appare all'orizzonte degli eroi greci. E questi eroi si sottopongono a dissidi tragici esattamente come i personaggi della modernità. Come dice Thomas Mann a Kerényi in una famosa lettera del 1941, la mitologia incontra la psicologia.

Proviamo a vedere come si presenta il nodo tra libertà e necessità in alcuni dialoghi, soprattutto là dove il personaggio è messo di fronte alle forze attrattive del mito. Nel primo dialogo composto da Pavese, Le streghe, dove parlano Circe e Leucotea, i termini sono già presenti, anche se poi la loro combinazione varierà da dialogo a dialogo. Circe, parlando degli uomini, dice che la morte li attira, e anche se morire è un destino, una ripetizione, un cosa saputa, essi si illudono che cambi qual- 
cosa. Nella prospettiva della dea, Odisseo è l'uomo che ha cercato di modificare il suo orizzonte entrando in contatto con il divino, senza rendersi conto che la dea era la depositaria di una sapienza immodificabile. Mentre possiede Circe, Odisseo è l'uomo in lotta con il proprio destino: «Era come una lotta con me, con la sorte. Voleva chiamarmi, tenermi, farmi mortale. Voleva spezzare qualcosa» (Pavese 1968a: 114).

Ma la soluzione di Odisseo non è l'unica prospettata da Pavese. Tutti sappiamo che nel secondo dialogo composto, La belva, Pavese ha profilato nelle parole di Endimione qualcosa che lo toccava da vicino. Salendo sul monte Latmo Endimione ha conosciuto le forme del divino e del bestiale e non può più sottrarsi agli effetti di quella conoscenza. Artemide gli è apparsa come una «cosa selvaggia, intoccabile, mortale, fra tutte le cose selvagge» (Pavese 1968a: 40), come la natura, cioè l'organo sessuale femminile, «di una lupa, di una daina, di una serpe». Per questo Endimione vuole ritrovare la dea, «esserle sangue sparso innanzi, essere carne nella bocca del suo cane» (Pavese 1968a: 42). Vuole cioè annullarsi, e il suo interlocutore, lo Straniero, gli spiega che allora deve addormentarsi senza svegliarsi più. Anche se così Pavese coglie in modo fortemente suggestivo un'ansia di dissolvimento dell'umano nel bestiale e nel divino (e quindi un'ansia di regressione), altri dialoghi aprono soluzioni diverse. Orfeo, nell'Inconsolabile, è, all'opposto di Endimione, l'eroe che si sottrae ai richiami del sesso, dell'ebbrezza e del sangue (i tre termini usati esplicitamente da Pavese nella premessa al dialogo), cioè a «beatitudini ctonie». Orfeo rifiuta la festa dionisiaca di cui gli parla la Baccante sua interlocutrice, e lucidamente vede nell'ebbrezza solo l'illusione che l'uomo può concepire per rompere il proprio destino. Voltandosi e perdendo Euridice, Orfeo si emancipa da una logica di ripetizione mitica. Nel barlume di cielo che gli compare all'uscita dall'Ade ha intravisto un'ipotesi di consapevolezza, un destino «più profondo del sangue, di là da ogni ebbrezza». Ha rifiutato cioè l'elemento mitico che Euridice incarnava e ha scelto il dàimon tragico, la logica che pone la morte e il nulla al termine di ogni esistenza.

Nel dialogo Il lago Ippolito, il giovane cacciatore ucciso da Afrodite ma reso divino da Diana col nome di Virbio, si pone in posizione opposta rispetto a Endimione ma anche rispetto a Orfeo. Diana lo ha collocato in un luogo fuori dal tempo, nel «vivo crepuscolo di un mattino perenne», ma il giovane sente di aver perso una parte essenziale di sé e di esser diventato «un'ombra tra le ombre degli alberi» e di passare «fra i tronchi e le cose come fossi una nuvola» (Pavese 1968a: 108). Quello che ora chiede a Diana è di tornare uomo, di «avere una voce e un destino». Ha bisogno di sentire di nuovo il sapore del sangue (cioè di cacciare animali) per poter calmare il suo sangue. Il sangue è il simbolo di una ricerca che esclude la perfezione divina. All'ansia regressiva di Endimione, che voleva spargere il proprio di fronte alla dea, risponde il desiderio di Virbio, che vuole ritrovare nel sangue il proprio destino di finitezza umana.

"Sangue" è in effetti una delle parole chiave di Pavese. Anche se il sangue per Pavese esiste solo nel momento in cui abbandona il corpo umano e bagna la terra. Potremmo pensare a un famoso componimento di Walt Whitman, dove il poeta desidera che il suo sangue si riversi su tutti gli oggetti del mondo per renderli interni alla 
sua poesia. Ma dobbiamo anche capire il rapporto che si instaura tra il sangue e la terra, cioè quel rapporto sacrale e rituale che Pavese trova illustrato da Frazer e che costituisce per lui una vera ossessione archetipica. Non è un caso che il termine venga alla luce con frequenza anche nel Mestiere di vivere lungo tutto il '44: «Il sangue è sempre versato irrazionalmente. Ogni cosa è un miracolo, ma nel caso del sangue lo si sente più acutamente, perché di là c'è il mistero» (7 febbraio '44; Pavese 2000: 274); «La natura ritorna selvaggia quando vi accade il proibito: sangue o sesso» (13 luglio; Pavese 2000: 284). La natura che sembra impassibile ritorna selvaggia (misteriosa) quando il sangue dell'uomo viene versato e la bagna, come se ci fosse una legge d'attrazione che li mette di nuovo in rapporto. Potremmo anche ipotizzare che i dialoghi, composti tra il ' 45 e il ' 46 , abbiano avuto la funzione di tenere sotto controllo il ritorno prepotente del tema del selvaggio nell'opera di Pavese.

Tra gli esempi di selvaggio (tragico) che nel diario (in data 2 settembre '44) Pavese indica di aver affrontato c'è una prosa di Feria d'agosto (composta due mesi prima, 21 giugno-3 luglio 1944) dove troviamo anticipati molti degli elementi che ho isolato all'interno dei dialoghi, proprio sullo sfondo di un'immagine che possiamo considerare come archetipo della palude (probabilmente Pavese non ha ancora letto la Philippson). Si tratta di Nudismo, dove un uomo racconta di una specie di vizio segreto, quello di prendere il sole nudo nascosto nella pozza che forma un torrente in una campagna solitaria. Si tratta di un rituale moderno sotto il quale si ripresenta la ricerca del rapporto con la terra e quindi una anticipazione del tema del bestiale. Ma questa ricerca procede a scatti, senza continuità, e al centro della prima parte della prosa c'è un vero conflitto tra il corpo dell'uomo e la natura che lo circonda e in cui si immerge. Stare nudo nella conca significa darsi alla natura che vorrebbe appropriarsi di quel corpo: «la natura non sopporta il nudo umano e con tutti i suoi mezzi si sforza, come fa coi cadaveri di appropriarselo» (Pavese 1968c: 160). Ma il corpo non può piegarsi a questo, e inizia un lungo processo di resistenza e offerta, una ripetizione che scandisce il tempo e crea l'impressione di una morte e di una rinascita continuamente alternate. Il selvaggio - come nota l'autore nel diario - «è ciò che ci manca», «ciò che non sappiamo». Anche se nel racconto si intravedono tracce di una metamorfosi bestiale: «L'acqua e il sole mi vanno facendo ogni giorno più fosco; credono così di cancellarmi, di coprirmi, ma non sanno che invece m'imbestiano» (Pavese 1968c: 161).

La natura viene assorbita in un processo erotico che potrebbe far pensare a una forma di panismo rovesciato, perché il corpo nudo non può annullare l'esistenza del mondo esterno ma anzi se ne fa cassa di risonanza: «Tutta quanta la conca cuoce al sole. Si sentono frulli, fruscii, tonfi, richiami che paiono venir da chi sa dove e non sono a tre passi. È in qualcuno di questi momenti che dimentico d'essere nudo. Chiudo gli occhi, e tutta quanta la campagna, le frutte, $i$ viottoli, le coste, i viandanti, riprendono di là dagli alberi esistenza e spazio, ogni cosa un sentore, un sapore, la sua realtà. Tutto va e viene intorno a me, che mi cuocio sull'erba» (Pavese 1968c: 161). Potremmo pensare che la prosa fin qui contenga un'anticipazione, realistica e non mitica, del desiderio di annientamento nel bestiale che abbiamo trovato nei dialoghi (il nudista senza nome come anticipatore di Endimione). Ma in realtà la seconda parte della prosa rivela che questo rituale di immersione nella terra ha un aspet- 
to che non si risolve nel piacere di regressione. Il personaggio manifesta il bisogno di essere visto dai suoi simili. Deve in qualche modo esibire il suo coraggio di nudità di fronte ad altri, che non siano però i borghesi che conosce e che non hanno la sua stessa sfrontatezza (delle donne che portano il costume estivo dice ironicamente che «si credono nude perché cambiano d'abito»). Passando vicino ai contadini che mietono sente invece una forma di simpatia. Il grano raccolto ha il colore scuro del suo corpo (qui Pavese sfiora il motivo sacrificale di origine frazeriana). È infatti una contadina la prima che lo scopre e gli passa vicino senza provare però nessuna meraviglia. Ed è poi un contadino che condivide con lui l'esperienza di un bagno nudi, in una scena piena di ambiguità. A questo punto il sentimento che si rivela non è più di comunione con la natura ma di inappartenenza alla campagna. Alla gioia che si accompagnava all'imbestiamento segue ora il dubbio. La terra in realtà è vestita, cioè lavorata dai contadini («lavorare è vestire la terra»), e il nudismo diventa un ozio, o peggio ancora una colpa, non più un atto di coraggio. Il sudore del lavoro copre la terra, mentre l'uomo si rifugia nella conca dove il suo sudore non è lavoro ma svago, vizio segreto. E allora quella che sembra ricerca di vita nella natura mostra il suo aspetto mortuario, funebre: «Io ogni giorno ci trovo la vita, ma poi mi stendo, corpo nero, come un morto» (Pavese 1968c: 165). Pavese ha tenuto insieme così regressione e crisi della presenza, il dramma dell'uomo primitivo indagato da De Martino nel Mondo magico (1948).

Anche in questo caso non c'è riscatto nel mito, ma profondo turbamento e ansia di fronte alla propria solitudine. Il nudismo diventa ferita esistenziale, segno di diversità. Possiamo anche paragonare questo frammento a una famosa scena della tetralogia di Giuseppe di Thomas Mann, a cui probabilmente Pavese pensava. Si tratta dei capitoli in cui i fratelli spingono Giuseppe nella fossa per eliminarlo. Anche Giuseppe viene privato dei vestiti e gettato in una cisterna. E Giuseppe sa di dover morire: «La fossa era profonda, e una salvezza, un ritorno alla vita anteriore alla caduta in quella profondità, non era neppur concepibile». Ma il modello mitico dell'astro che tramonta e risorge offre a Giuseppe la speranza della rinascita: «l'idea della morte dell'astro, dell'oscuramento del figlio, del suo precipitare nel mondo sotterraneo che diventava sua dimora, includeva l'idea della nuova luce, della riapparizione e della resurrezione». Il mito in Mann agisce come fondamento narrativo e come componente psicologica del personaggio. Giuseppe rinascerà sotto altra forma. Sarà partorito di nuovo dalla terra. Il mito fa parte del suo destino.

Per Pavese invece l'immersione nella pozza non comprende un riscatto mitico, né tanto meno può funzionare come base narrativa dal momento che il racconto non ha vere scansioni e si riavvolge di continuo su se stesso. Ma il frammento verrà di nuovo utilizzato dentro il più complesso sistema del Diavolo sulle colline, dove il coraggio del nudismo viene vissuto dai tre amici come ricerca esplicita di un rapporto con la natura. Anche in questo caso si tratta di entrare nella terra per trovare se stessi, come Orfeo che scende nell'Ade e diventa consapevole del nulla. Ora Pavese ha distribuito tutte le componenti simboliche dell'immagine mitica tra $i$ tre amici, il narratore, Pieretto e Oreste. È Pieretto a enunciare l'idea per cui la campagna d'estate è imbevuta di morte, «tutto vive e si macera in se stesso. La natura è la morte...», che per il narratore diventa uno dei motivi conduttori dell'avventura. E 
sono Pieretto e Oreste a condurlo al pantano, il luogo dove si consuma il rito di imbestiamento nella terra: «Quel brivido di starcene nudi e saperlo, di nasconderci a tutti gli sguardi, e bagnarci, annerirci come tronchi, era qualcosa di sinistro: più bestiale che umano. Scorgevo nell'alta parete dello spacco affiorare radici e filamenti come tentacoli neri: la vita interna, segreta della terra» (Pavese 1968b: 130).

Ma il narratore sente anche che la terra sta entrando in lui attraverso i cibi da lei prodotti, e che il suo sangue si intride del sangue della campagna. In altre parole, è lui a provare più intensamente il rischio dell'imbestiamento, ma nello stesso tempo è lui che riesce più degli altri a tenersene al riparo. Il dubbio è la sua arma di difesa. Mentre il cinico Pieretto non crede in niente, e non ha paura, mentre Oreste si fa coinvolgere in un'avventura amorosa impossibile con Gabriella, e Poli è ormai schiavo di Dioniso (cioè dell'alcol e delle droghe), il narratore elabora, con un progressivo isolamento, una sua personale salvezza. Attraverso l'esperienza della palude viene a contatto con la vita della terra, ma come Asclepio, il mitico personaggio nato da Corònide, riesce ad attraversarla, e ad amare la selvaggia collina del Greppo. In lui c'è il benefico influsso di Hermes-Mercurio, lo stesso dio che Mann invocava come scudo di fronte all'irrazionale. Come Hermes, egli può stare nella profondità della palude $\mathrm{e}$ risalire sulla collina. Come Hermes, può sperimentare in sé la contraddizione ${ }^{2}$. E la collina, il luogo dell'incanto diabolico, gli si presenta come mondo del lavoro e della fatica (anche questo un carattere ermetico). Ma la stessa collina non gli consente di diventare radice, cioè di trovare appartenenza in essa, e così la sua avventura resta tragicamente sospesa (anticipando la sospensione della ricerca di Anguilla). Alla fine, quando l'estate declina e si deve lasciare il Greppio, il narratore dichiara apertamente di non volersi voltare a guardarlo. Il tragico, come vuole Pavese in una lettera, compare qui come «lievissima incrinatura» nella salute umana.

Concludo. Una palude di sangue e una collina (oltre che una vigna) sono iscritti nell'opera di Pavese. Si tratta del paesaggio della Tessaglia descritto dalla Philippson. Sul monte Elicona Esiodo ascolta dalla musa il racconto della palude. Sulle colline del Piemonte i personaggi degli ultimi romanzi scoprono il selvaggio calato tragicamente nel quotidiano. Anche se Calvino costringe il suo personaggio più mercuriale, l'inafferrabile Qfwfq, ad abbandonare la palude delle origini (è l'esito richiesto dalla virtù della leggerezza), non possiamo ignorare che la modernità incarnata da Pavese prevede invece la persistenza del mito nel tragico. Calvino nasce da una costola di Pavese. Hermes è figlio di Boibeide.

${ }^{2}$ Cfr. Frontaloni (2005: 111): «A differenza di Dioniso, che 'porta in sé e da sé rivela i dissidi della realtà', il messaggero degli dei, proprio in virtù del suo ruolo di mediatore fra mondo titanico e mondo olimpico, è infatti colui che 'crea in sé la contraddizione'». 


\section{Bibliografia}

De MARTINO, Edoardo (1948): Il mondo magico: prolegomeni a una storia del magismo.Torino, Einaudi.

FRONTALONI, Elena (2005): «Dare un nome, rivelare un dio. Extase, formula e tempo nei Dialoghi con Leucò di Cesare Pavese», in La Cultura, XLII, 1, aprile 2005, pp. 95-130.

GugliELMI, Guido (1998): «Pavese mitologo», in La prosa italiana del Novecento II. Tra romanzo e racconto. Torino, Einaudi.

KerÉNYI, Károly, in Jung, Carl. G. - KERÉNYI, Károly (1971): Prolegomeni allo studio scientifico della mitologia, trad. it. di A. Brelich. Torino, Bollati Boringhieri.

PAVESE, Cesare (1968a): Dialoghi con Leucò, a cura di Sergio Givone. Torino, Einaudi.

PAVESE, Cesare (1968b), Il diavolo sulle colline, in La bella estate. Torino, Einaudi.

PAVESE, Cesare (1968c), Nudismo, in Feria d'agosto. Torino, Einaudi.

PAVESE, Cesare (2000), Il mestiere di vivere, a cura di Marziano Guglielminetti e Laura Nay. Torino, Einaudi.

PHILIPSON, Paula. Thessalische Mythologie, in Origini e forme del mito greco (1949), trad. it. di A. Brelich. Torino, Einaudi.

UntesteIner, Mario (1972): Fisiologia del mito. Firenze, La Nuova Italia. 